\title{
Evidence that polyploidy in esophageal adenocarcinoma originates from mitotic slippage caused by defective chromosome attachments
}

\author{
Stacey J. Scott ${ }^{1} \cdot$ Xiaodun $\mathrm{Li}^{2}$ - Sriganesh Jammula ${ }^{3}$ - Ginny Devonshire ${ }^{3} \cdot$ Catherine Lindon $^{4}$. \\ Rebecca C. Fitzgerald $\mathbb{( i D}^{2} \cdot$ Pier Paolo D'Avino $\mathbb{B}^{1}$
}

Received: 2 July 2020 / Revised: 22 January 2021 / Accepted: 28 January 2021 / Published online: 1 March 2021

(c) The Author(s) 2021. This article is published with open access

\begin{abstract}
Polyploidy is present in many cancer types and is increasingly recognized as an important factor in promoting chromosomal instability, genome evolution, and heterogeneity in cancer cells. However, the mechanisms that trigger polyploidy in cancer cells are largely unknown. In this study, we investigated the origin of polyploidy in esophageal adenocarcinoma (EAC), a highly heterogenous cancer, using a combination of genomics and cell biology approaches in EAC cell lines, organoids, and tumors. We found the EAC cells and organoids present specific mitotic defects consistent with problems in the attachment of chromosomes to the microtubules of the mitotic spindle. Time-lapse analyses confirmed that EAC cells have problems in congressing and aligning their chromosomes, which can ultimately culminate in mitotic slippage and polyploidy. Furthermore, whole-genome sequencing, RNA-seq, and quantitative immunofluorescence analyses revealed alterations in the copy number, expression, and cellular distribution of several proteins known to be involved in the mechanics and regulation of chromosome dynamics during mitosis. Together, these results provide evidence that an imbalance in the amount of proteins implicated in the attachment of chromosomes to spindle microtubules is the molecular mechanism underlying mitotic slippage in EAC. Our findings that the likely origin of polyploidy in EAC is mitotic failure caused by problems in chromosomal attachments not only improves our understanding of cancer evolution and diversification, but may also aid in the classification and treatment of EAC and possibly other highly heterogeneous cancers.
\end{abstract}

Edited by G. Melino

Supplementary information The online version contains supplementary material available at https://doi.org/10.1038/s41418021-00745-8.

$\triangle$ Pier Paolo D’Avino

ppd21@cam.ac.uk

1 Department of Pathology, University of Cambridge, Cambridge, UK

2 Medical Research Council Cancer Unit, Hutchison/Medical Research Council Research Centre, University of Cambridge, Cambridge, UK

3 Cancer Research UK Cambridge Institute, University of Cambridge, Cambridge, UK

4 Department of Pharmacology, University of Cambridge, Cambridge, UK

\section{Introduction}

Genomic instability drives evolution, diversification, heterogeneity, and adaptation in many cancers. One type of genomic instability, chromosomal instability (CIN), promotes large scale structural and numerical genomic changes that can lead to punctuated evolution by producing abrupt changes in the balance of critical growth and death pathways [1]. CIN is usually classified as either structural-to indicate alterations in chromosome structure, such as translocations, inversions, duplications-or numericalcharacterized instead by recurrent gain or loss of chromosomes that lead to aneuploidy and/or polyploidy. Some cancers present one specific type of CIN, but the two can occur together and numerical CIN can subsequently lead to structural chromosomal aberrations. Although both aneuploidy and polyploidy are very common in various cancers and numerical CIN is now considered a key factor in cancer evolution and diversification, its origin and exact role in cancer onset are still debated $[1,2]$. There is evidence that the presence of extra centrosomes, which is observed in 
various cancers, can directly lead to an increase in incorrect (merotelic) attachments of chromosomes to the spindle microtubules that in turn cause chromosome missegregation and aneuploidy [3, 4]. By contrast, our knowledge of the mechanisms that trigger polyploidy in cancer cells is still poor, despite the evidence that nearly $30 \%$ of different cancer types present whole-genome doubling (WGD) events and that polyploidy propagates CIN, accelerates cancer genome evolution, increases tolerance to chromosome mis-segregation and drug treatments, and is associated with poor cancer prognosis [5-8].

Esophageal adenocarcinoma (EAC), the predominant histological type of esophageal carcinomas in the western world with high mutation burden and substantial heterogeneity [9-12], represents an ideal system to study the origins and role of polyploidy in cancer evolution and heterogeneity. EAC develops from a pre-cancerous condition known as Barrett's esophagus (BE). BE can progress from a non-dysplastic lesion through intermediate stages of low-grade and high-grade dysplasia leading to EAC formation [13]. During EAC development, the copy number and heterogeneity of the genome increases and the spectrum of mutations and rearrangements shows very little overlap with its paired BE counterpart [14]. Whole-genome sequencing (WGS) of paired BE and EAC samples indicated that, although approximately $80 \%$ of point mutations found in EAC samples are already present in the DNA from the adjacent BE epithelium [15], the difference in copy number aberrations between BE vs. EAC samples was much more dramatic. BE samples contained very few copy number changes and were mostly diploid, whereas EAC showed a wide range of copy numbers changes including some highly amplified regions $[14,16]$. These studies also found that up to two-thirds of EACs emerged following a WGD event (tetraploidy), in a proposed pathway comprising an initial loss of p53 followed by tetraploidy and subsequent CIN [16].

There are four events that, in principle, can lead to polyploidy: cell fusion, genome endoreduplication, cytokinesis failure, or mitotic slippage. In the latter case, cells fail to satisfy the mitotic checkpoint and, after sustaining a prolonged arrest, the chromosomes decondense without segregating to the poles and cells re-enter in G1 phase. Both cytokinesis failure and mitotic slippage result in the formation of cells with a polyploid number of chromosomes, but the first leads to cells containing two equally sized nuclei (binucleate) whereas the second generally leads to cells with a single, large tetraploid nucleus. As no clear evidence for either cell fusion or endoreduplication has been reported in BE and EAC, we hypothesized that genome doubling in EAC development might arise as a result of a defect in cell division. To address this hypothesis and to understand the origin(s) of polyploidy in EAC, we analyzed cell division in both a 2D cell system that recapitulate EAC development and in patient-derived organoids. Our findings indicated that polyploidy in EAC originates from mitotic slippage caused by failure in chromosome alignment and segregation. Furthermore, WGS, RNA-seq, and quantitative immunofluorescence analyses suggested that an imbalance in the amount, and possibly regulation, of proteins involved in the attachment of chromosomes to spindle microtubules could be the molecular mechanism underlying this mitotic failure.

\section{Results}

\section{p53-deficient BE and EAC cells display specific mitotic defects}

As a first step to investigate the potential origin(s) of polyploidy in EAC, we analyzed cell division in a panel of $\mathrm{BE}$ and EAC cultured cells specifically selected in order to recapitulate the stages of progression from the premalignant condition to the carcinoma. We used two BE cell lines: CPA and CPD; the first is non-dysplastic, neardiploid, and has wild-type p53, while the second is dysplastic, near tetraploid, and have mutated p53. We also analyzed four different EAC cell lines (all near-tetraploid and p53-deficient) and included the non-transformed immortalized RPE-1 cell line as a reference control cell line (Supplementary Table S1). To analyze mitosis, cells were stained by immunofluorescence for the mitotic marker histone $\mathrm{H} 3 \mathrm{pS} 10$, tubulin, and DNA, to calculate the frequency of mitotic cells or mitotic index (MI) and visualize the mitotic spindle and chromosomes. All BE and EAC cells had an MI lower than the control RPE1 cells (Fig. 1a). CPA, CPD, and FLO cells had very similar MI values at around 4\%, whereas JH-Eso-Ad1 and OE19 had slightly lower MIs, and OE33 cells had the highest MI among the esophageal cell lines, which was comparable to that of RPE1 cells (Fig. 1a). Quantification of cells at different mitotic stages (prophase/prometaphase, metaphase, anaphase, and telophase/cytokinesis) revealed some interesting differences (Fig. 1b, Supplementary Table S2). As expected, in control RPE-1 cells the highest percentage of mitotic cells were found to be in prophase/prometaphase and telophase/cytokinesis (Fig. 1b). A similar trend was observed in the two BE cell lines and in OE19, albeit both CPA and CPD had a low percentage of cells in telophase/cytokinesis (Fig. 1b). By contrast, the other three EAC cell lines had a higher percentage of cells displaying a metaphase plate with apparently aligned chromosomes compared to RPE-1 and BE cells, which in FLO and JH-Eso-Ad1 was even higher than the number of cells in prophase. The combination in these EAC cells of an increase in metaphase cells without a 
a

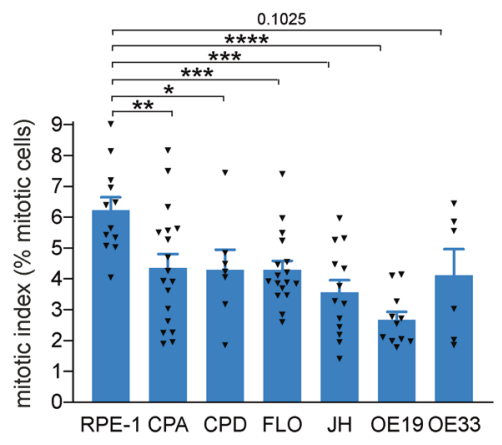

C
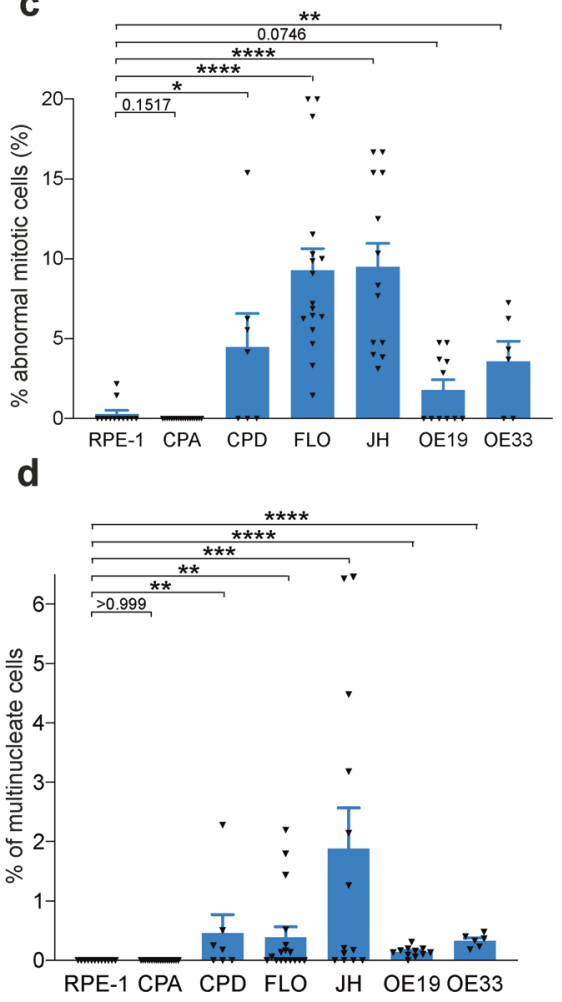

g

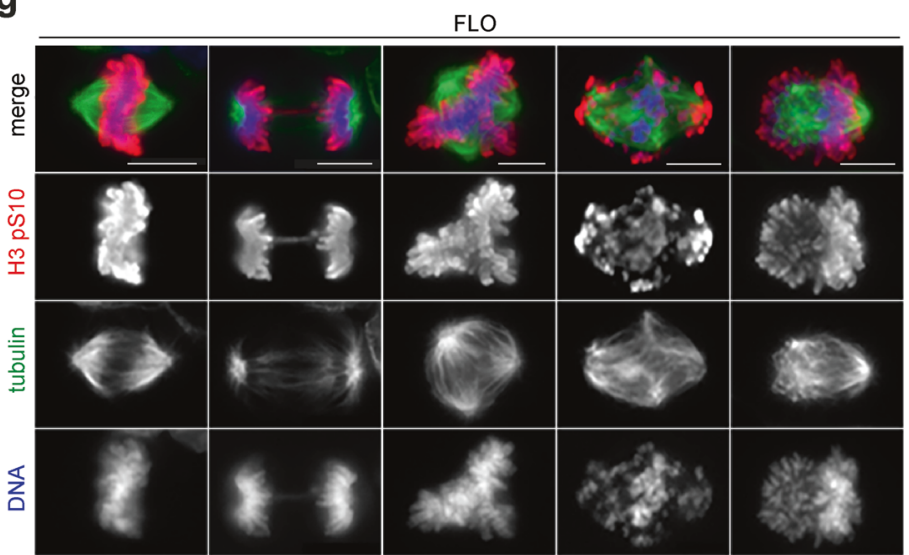

metaphase b
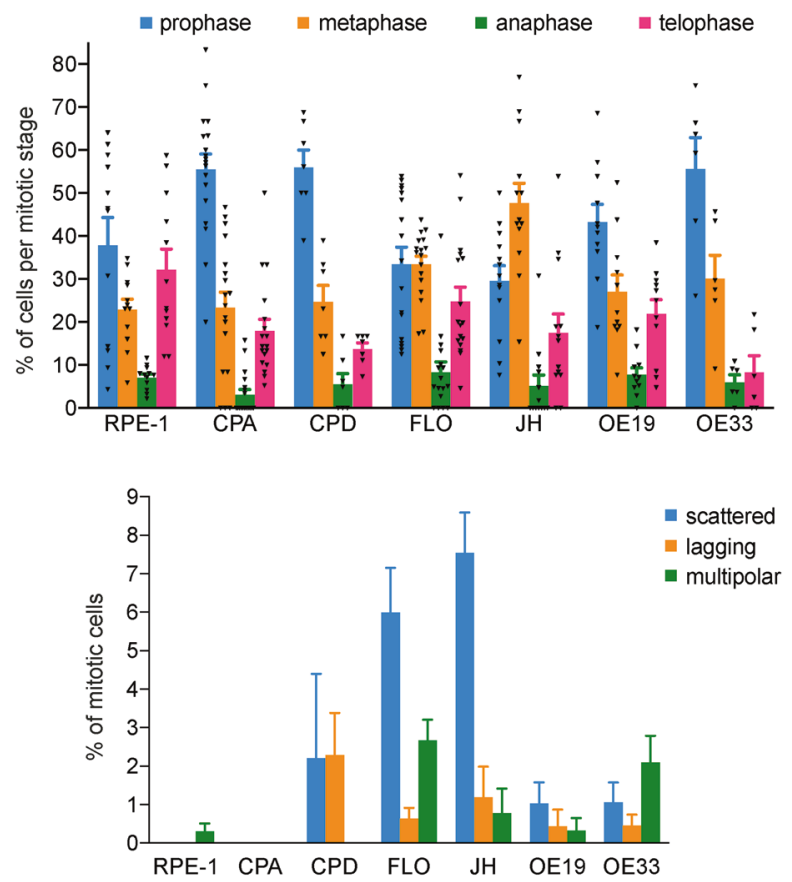

f

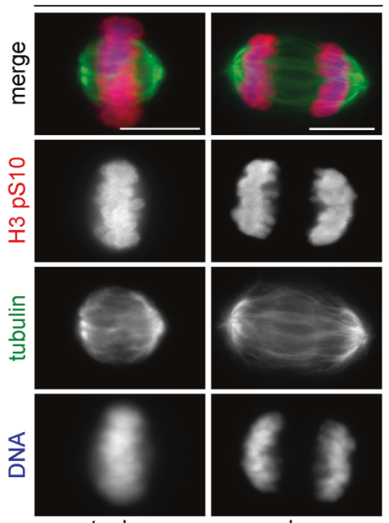

metaphase

anaphase

metaphase
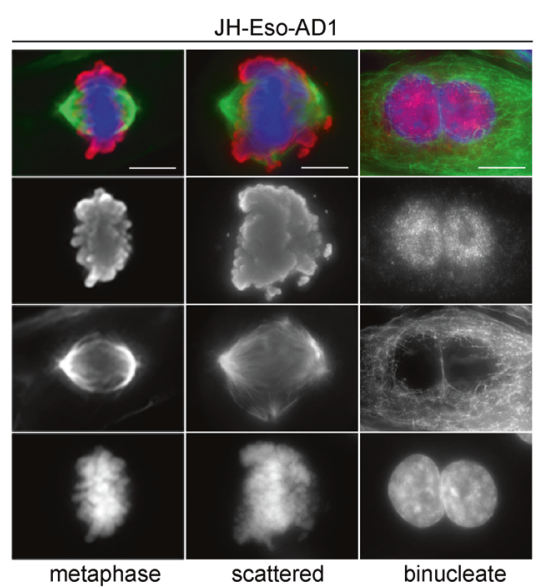

high MI indicates that they spend long time in a metaphaselike configuration, possibly because of problems in aligning and/or segregating their chromosomes, but can then successfully progress through mitosis and the rest of the cell cycle. Immuno-fluorescence analysis also revealed that the p53-deficient CPD and EAC cell lines displayed a higher 
Fig. 1 BE and EAC cells display specific mitotic defects. a Cells from representative $\mathrm{BE}$ and $\mathrm{OAC}$ cell lines were stained to detect the mitotic marker histone $\mathrm{H} 3 \mathrm{pS} 10$, tubulin and DNA (see $\mathbf{f}$ and $\mathbf{g}$ below). The percentages of cells in mitosis (mitotic indices) were counted (a) and categorized by each mitotic stage (b) through visual analysis of the presence of $\mathrm{H} 3 \mathrm{pS} 10$ and characteristic mitotic figures. In addition, for each cell line the number of abnormal mitoses was also counted (c) and categorized into one of three phenotypes: lagging chromatin, multipolar spindles, or scattered chromosomes (e). d Graph showing the percentages of multinucleate cells from the experiments described in $(\mathbf{a}-\mathbf{g})$. More than 3000 cells in total and more than 200 mitotic cells per each cell line were counted; $n \geq 6$ independent experiments; ${ }^{*} p<$ $0.05, * * p<0.01, * * * p<0.001, * * * * p<0.0001$ (Mann-Whitney $U$ test). Two-way ANOVA statistical analyses with multiple comparisons of the data in (b) and (e) are shown in Supplementary Table S2 and S3, respectively. To improve visualization, only the summary of the data is shown in (e), but a similar graph including also the individual values is shown in Supplementary Fig. S1. In each graph, bars indicate SEM. f, g Representative images from the indicated $\mathrm{BE}$ and $\mathrm{OAC}$ cell lines fixed and stained to detect the mitotic marker histone $\mathrm{H} 3 \mathrm{pS} 10$ (red in the merged images), tubulin (green in the merged images), and DNA (blue in the merged images). Bars, $10 \mu \mathrm{m}$.

frequency of mitotic defects (up to 10\%) than RPE-1 and CPA cells (Fig. 1c). A similar result was observed for the quantification of multinucleate cells (cells containing two or more equally sized nuclei; a readout for cytokinesis failure), although the frequency of this phenotype was much lower $(<2 \%)$ (Fig. 1d). The mitotic defects were categorized into three main phenotypes: lagging chromatin/chromosomes, multipolar spindles, and defects in chromosome congression, characterized by the presence of misaligned chromosomes (Fig. 1e-g, Supplementary Fig. S1, Supplementary Table S3). Chromosome congression defects presented with varying levels of severity; in some cases most chromosomes successfully aligned at the metaphase plate with just a few uncongressed chromosomes, whereas in other severe cases all chromosomes failed to congress and were randomly distributed over the mitotic spindle (Fig. 1f, g). We will hereafter collectively refer to chromosome congression defects as the "scattered chromosomes" phenotype. This phenotype was one of the most frequently observed across all the p53-deficient cell lines and was absent in both RPE-1 and CPA.

A previous study reported that centrosome amplification occurred early in the progression of BE into EAC, and that this was dependent upon p53 loss [17]. As supernumerary centrosomes can cause mitotic defects, we analyzed their presence in our BE cell lines and in the two EAC cell lines, FLO and JH-Eso-Ad1, that had the highest percentage of mitotic defects (Fig. 1e). We stained BE and EAC cells with antibodies against Plk4 and $\gamma$-tubulin to mark and quantify centrioles and centrosomes, respectively, and assessed whether extra centrosomes correlated with multipolar spindles and/or scattered chromosomes (Supplementary Fig. S2). Both BE cell lines showed only bipolar spindles with two centrosomes and correctly aligned chromosomes, while FLO and JH-Eso-Ad1 cells had 10-12\% of cells with more than two centrosomes (Supplementary Fig. S2b), which often generated multipolar spindles, but with properly congressed chromosomes (Supplementary Figs. S2a and S2c). Importantly, scattered chromosomes were only observed in cells with two centrosomes and bipolar spindles (Supplementary Fig. S2c).

Together, our results indicate that p53-deficient BE and EAC cells have a significant increase in cells with misaligned chromosomes that do not appear to be associated with extra centrosomes and multipolar spindles.

\section{EAC cells have a functional spindle assembly checkpoint and manifest mitotic slippage}

We next employed time-lapse microscopy to better understand the origin of the mitotic defects in both BE and EAC cells and how they affected progression through mitosis. However, we first established whether these cell lines had a functional spindle assembly checkpoint (SAC), the surveillance mechanism that prevents mitotic exit until all chromatids have correct bipolar attachments [18]. In the presence of a functional SAC, cells arrest in mitosis when treated with the microtubule depolymerizing drug nocodazole. BE, EAC, and RPE-1 cells displayed variable increases in MI after nocodazole treatment, clearly indicating that they all possess a functional SAC (Supplementary Fig. S3).

We incubated CPA, FLO, and JH-Eso-Ad1 cells with the SiR-DNA dye to visualize chromosomes and then recorded images at 5-min intervals for $8-10$-h periods to monitor their progression through mitosis (Fig. 2). As expected, almost all CPA cells $(90.0 \% ; n=30)$ progressed through mitosis without problems (Fig. 2A, Supplementary Movie 1) and only a small percentage of CPA cells showed lagging chromatin or failed cytokinesis (Fig. 2E). Similar to the results observed in fixed cells, $72 \%$ of FLO cells divided normally, while $6 \%$ formed multipolar spindles, $11 \%$ lagging chromatin (Fig. 2B, Supplementary Movie 2), and $11 \%$ experienced mitotic slippage (Fig. 2E). In the last case, cells formed a broad metaphase plate, indicating chromosome congression defects, spent up to $3 \mathrm{~h}$ in this configuration without entering anaphase, and then the chromosomes appeared to decondense and a nucleus reformed (Fig. 2C, Supplementary Movie 3). Mitotic slippage was also observed with even higher frequency in $\mathrm{JH}$ Eso-Ad1 cells $(28.6 \% ; n=7$ ) (Fig. 2D, E). Cells that failed to progress past metaphase appeared to have congressed their chromosomes at the equator, forming a well-defined metaphase plate, 30 min after nuclear envelope breakdown (NEB), which marks the beginning of prometaphase. The cells then remained in this phase for about 90 min until the 
A

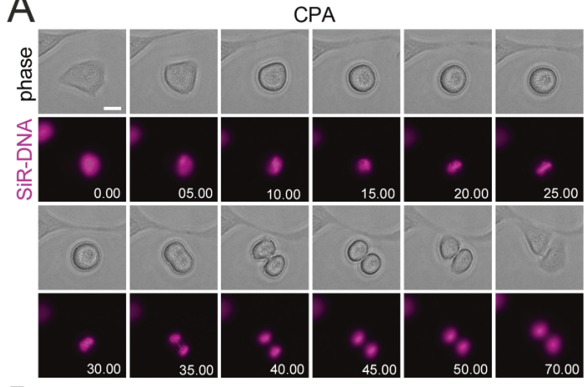

B

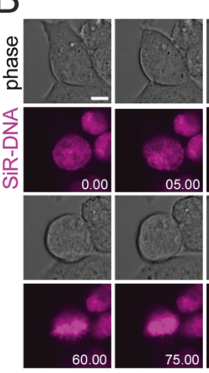

$E$

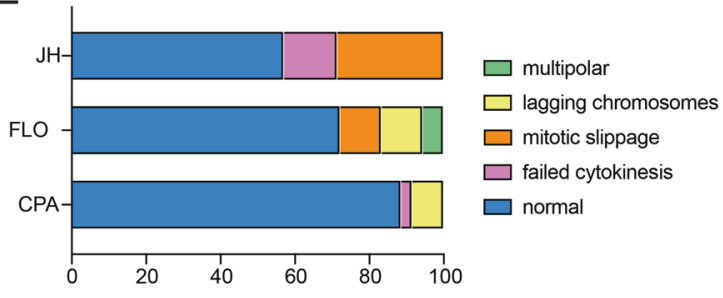

$\mathrm{D}$

C
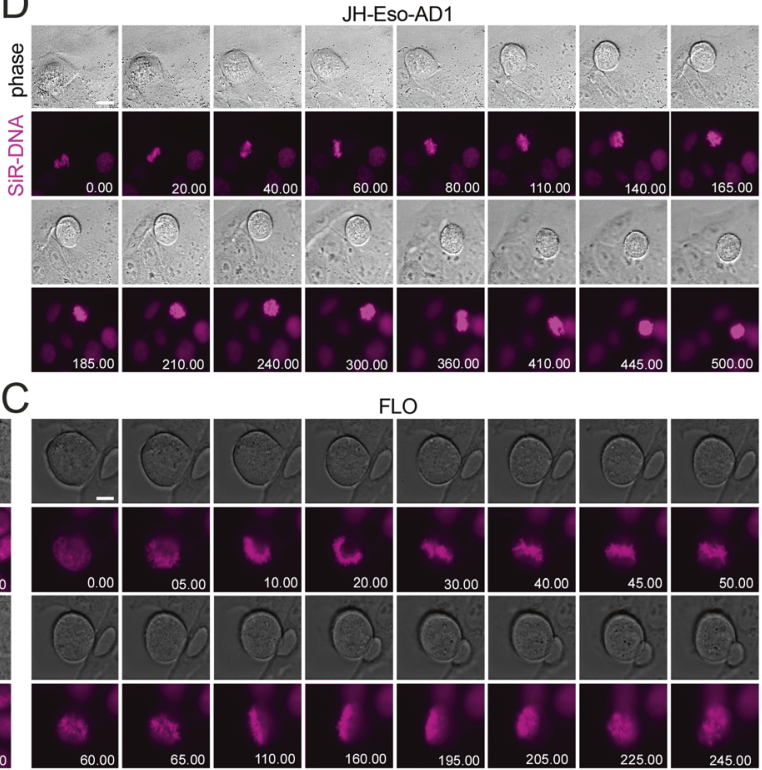

$\mathrm{F}$

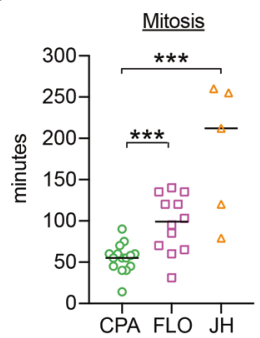

Fig. 2 EAC cells have problems in congressing and aligning chromosomes and display mitotic slippage. A-D Images from timelapse recordings of the indicated $\mathrm{BE}$ and EAC cells treated with SiRDNA dye to visualize chromosome dynamics. Images were captured at $5 \mathrm{~min}$ intervals for $8-10 \mathrm{~h}$. Time is in min:sec relative to nuclear envelope breakdown (NEB). The arrows in (B) mark lagging chromatin. Bar, $10 \mu \mathrm{m}$. E Graph showing the frequency of phenotypes

chromosomes began to drift away from the metaphase plate. In extreme cases, like the one showed in Fig. 2D, $6 \mathrm{~h}$ after NEB the chromosomes drifted from the metaphase plate and attempted to realign, but failed and by the end of the recording period $(8 \mathrm{~h})$ the cell begun to decondense the chromosomes, possibly because of cohesion fatigue (Fig. 2D, Supplementary Movie 4). The number of filmed JH-Eso-Ad1 cells was lower than the other two cell lines because most of the cells died during or prior to filming, despite our extensive troubleshooting to establish optimal conditions for these cells.

Our time-lapse experiments allowed us also to calculate the length of mitosis and we found that EAC cells took at least twice as long to divide than BE cells. Mitosis in CPA cells was completed in about $1 \mathrm{~h}(58 \pm 14 \mathrm{~min})$, while it took an average of $103( \pm 31)$ min in FLO cells and $212( \pm 19)$ min in JH-Eso-Ad1 cells (Fig. 2F). It took also significantly longer for FLO and JH-Eso-Ad1 cells to reach anaphase onset after NEB than CPA cells (Fig. 2F), indicating that observed in the time-lapse recordings described in (A-D). 30 independent CPA cells, 18 independent FLO cells, and 7 independent JHEso-AD1 cells were analyzed. F Scatter plots showing quantification of the length of mitosis (from NEB until telophase) and the length of prometaphase-anaphase (from NEB until anaphase onset). $n=15$ for CPA cells, $n=12$ for FLO cells, and $n=5$ for JH-Eso-AD1 cells; $* * * p<0.001, * * * * p<0.0001$ (Mann-Whitney $U$ test).

chromosome congression was more challenging in EAC cells.

In conclusion, these findings indicate that EAC cells have problems in congressing and aligning chromosomes, which can ultimately cause mitotic slippage and polyploidy.

\section{EAC organoids display mitotic defects similar to cultured cells}

EAC cell lines are the most widely used model for EAC research, but in many cases we lack genomic information about both their primary tumors and germlines [19]. Moreover, they can only recapitulate EAC to an extent because culturing them over numerous passages make them unrepresentative of the mutational features of the original tumor [19]. To overcome these limitations, we have recently develop a series of EAC-derived organoids that more faithfully represent the primary cancers from which they derived and can be stably maintained [20]. Therefore, we decided to further 


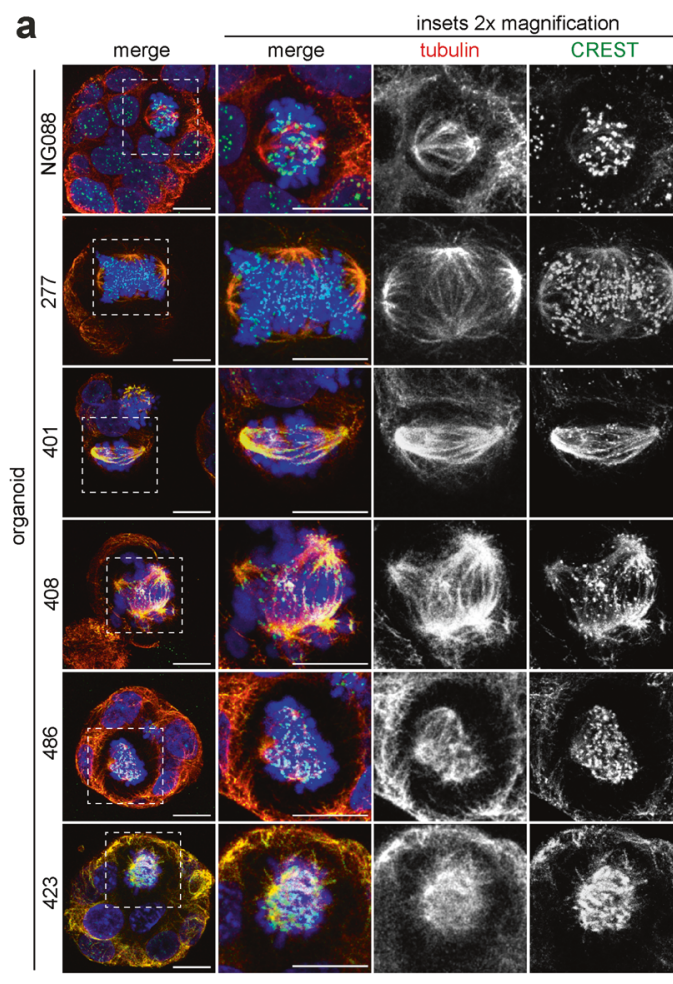

Fig. 3 EAC organoids display mitotic defects similar to EAC cultured cells. a The indicated control and EAC organoids were fixed and stained to detect tubulin (red in the merged images), the centromeric marker CREST (green in the merged images), and DNA (blue in the merged images). Bars, $10 \mu \mathrm{m}$. b Graph showing the quantification of

validate our findings from cultured cell lines by investigating whether EAC organoids presented similar mitotic defects.

A selection of organoids that represent healthy gastric tissues (NG088) and EACs with various ploidies and karyotypes (Supplementary Table S4 and Supplementary Fig. S4) were stained for tubulin, DNA, and the centromeric marker CREST [21] to visualize, characterize, and quantify mitotic figures (Fig. 3a). Almost all $(95 \%, n=20)$ mitotic spindles observed in a normal gastric organoid line (NG088) were bipolar and had correctly congressed chromosomes (Fig. 3a and b). By contrast, all EAC organoids presented a much higher frequency of abnormal mitoses than NG088 (Fig. 3b). Similar to the data collected from cell lines, the most prevalent mitotic aberrations observed in EAC organoids were multipolar spindles and scattered chromosomes (Fig. 3c). With the exception of organoid CAM423, multipolar spindles were present in at least $10 \%$ of mitoses. The incidence of scattered chromosomes (5-20\%) was sometime lower than multipolar spindles (Fig. 3c), but still comparable to the data obtained from EAC cell lines (Fig. 1). Interestingly, although these two mitotic defects were observed in both polyploid and aneuploid EAC organoids, one of the two polyploid organoids, CAM423, did not show multipolar spindles, while the other, CAM277, had no scattered chromosomes (Fig. 3c). b
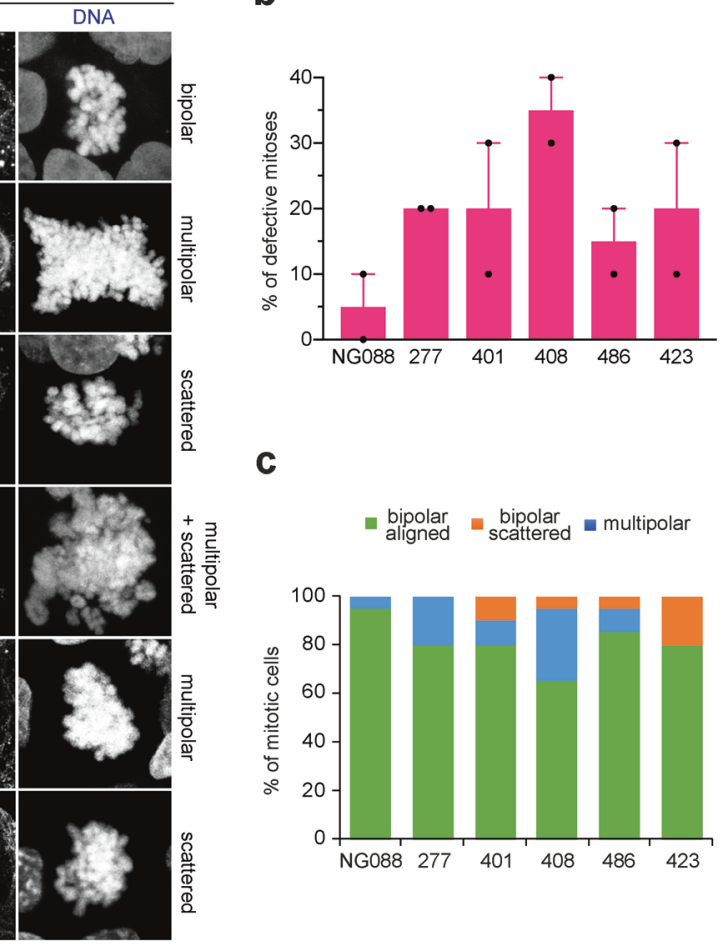

C

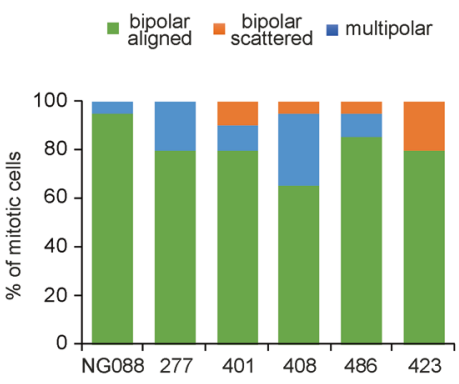

mitotic defects observed in the indicated organoids; 20 organoids in 2 independent experiments were analyzed for each group. c Graph showing the frequency of phenotypes observed in the organoids described in $(\mathbf{a}, \mathbf{b})$.

Together, our findings indicate that EAC organoids present the same types of mitotic aberrations observed in EAC and BE cell lines. Although we could not find any clear correlation between mitotic defects and ploidy, the presence of scattered chromosomes did not appear to depend on the formation of multipolar spindles, which is consistent with the cell line data.

\section{WGS of BE and EAC cell lines and primary tumors shows numerous copy number changes in kinetochore genes}

The presence of chromosome alignment defects in both EAC cells and organoids suggested that the attachment of chromosome to microtubules might be impaired in these cells. The centromeric regions of chromosomes attach to microtubules through the kinetochore, a macromolecular structure composed of a multitude of proteins and protein complexes [22]. The kinetochore is divided into two layers, the inner and outer kinetochore. The inner kinetochore comprises many CENP proteins that assemble onto the major centromeric protein CENP-A, a centromere-specific variant of histone $\mathrm{H} 3$, to form the constitutive centromereassociated network (CCAN) [22]. The outer kinetochore is comprised primarily of the large multi-subunit Knl1/Mis12/ 


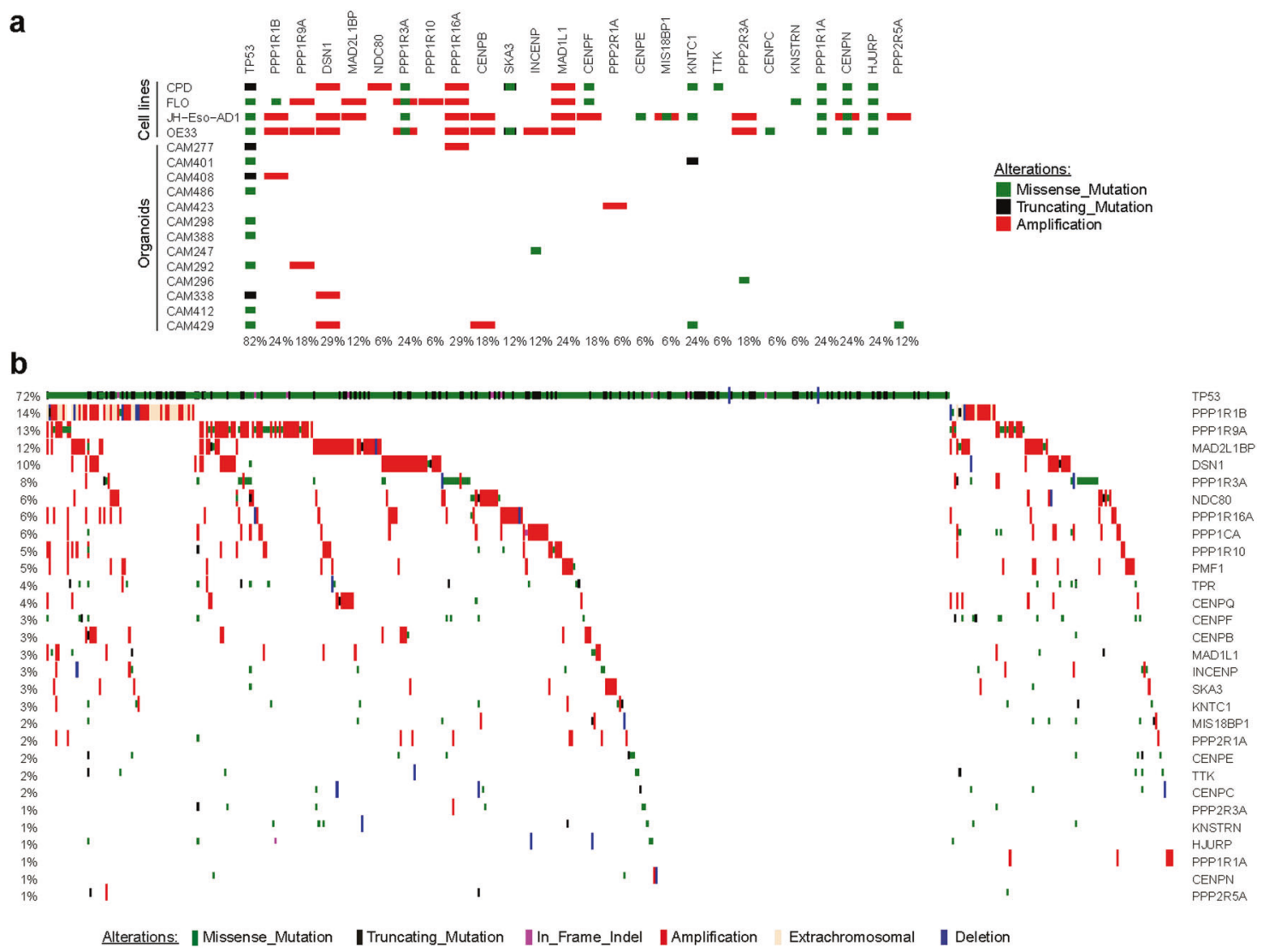

Fig. 4 Genes involved in chromosome attachments are altered in EAC cells, organoids, and tumors. Diagrams showing the frequency and nature of SNVs, indels, and copy number alterations of genes involved in chromosome attachment in cell lines and organoids (a),

Ndc80 complex network (KMN network), which is recruited by the CCAN at the inner kinetochore to form strong interactions with mitotic spindle microtubules [23]. Furthermore, the association of kinetochores to microtubules is finely regulated by phosphorylation mediated by serine/ threonine kinases and counteracting phosphatases, including Aurora B kinase (AURKB) [24].

We therefore investigated whether EAC cells, organoids, and tumors presented alterations in the structure and copy numbers of genes that might be responsible for the chromosome attachment defects observed in EAC cells and organoids. We selected genes encoding proteins known to be involved in chromosome alignment, including kinetochore components (e.g., CCAN and KMN proteins), SAC proteins, and regulatory factors, such as AURKB, Polo-like kinase 1 (Plk1), and members of the PP1 and PP2A phosphatase families [22, 25] (Supplementary Table S5). WGS analyses revealed a number of alterations in our selected gene set that were shared across cell lines, organoids, and tumors (Fig. 4). The most common feature was gene amplification, which was frequently observed for the outer kinetochore components and EAC cases $(n=379)$ from the Oesophageal Cancer Clinical And Molecular Stratification (OCCAMS) consortium (b). Total proportion of cases altered by individual gene are listed at the bottom (a) or at the left (b) of the oncoplot.

Dsn1 and Ndc80, the SAC proteins Mad1 and Mad2binding protein MAD2L1BP (also known as p31 comet) [26], and various members of the PP1 family of phosphatases (Fig. 4). The picture for CCAN inner centromeric proteins (CENPs) was more mixed, with a combination of amplifications and missense mutations (Fig. 4). RNA-seq analyses confirmed that, in tumors, gene amplification corresponded to a significant increase in gene expression levels $(p$ value $<0.0001)$ for the mostly frequently amplified genes encoding for Dsn1, MAD2L1BP, and the regulatory PP1 subunit PPP1R1B (Fig. 5a). In many cases, PPP1R1B was over-amplified (ploidy adjusted copy number $>10$ ), which is particularly interesting considering its close proximity to the oncogene ERBB2 that is also frequently over-amplified in EACs. Moreover, single sample gene set enrichment analysis showed a coenrichment of our gene signatures with known cell-cycle hallmark gene signatures (Fig. 5b).

These findings suggest that alterations in the copy number and expression of genes involved in chromosome attachment might be connected to the mitotic defects observed in both cell lines and organoids. 
Fig. 5 The expression of genes involved in chromosome attachments is altered in EAC tumors. a Graphs illustrating expression levels measured in log-transformed Transcripts Per Million (TPM) for genes PPP1R1B, DSN1, and MAD2L1BP across EAC cases with different alteration types showing significant increase in expression upon amplification as compared to control wild type (WT) cases. ( $p$ values computed through Wilcoxon test). b RNAseq based single sample geneset enrichment analysis of annotated gene signatures across EAC cases $(n=145)$ shows coenrichment of our kinetochore gene signature (Supplementary Table S5) with known cell-cycle hallmark gene signature [49].
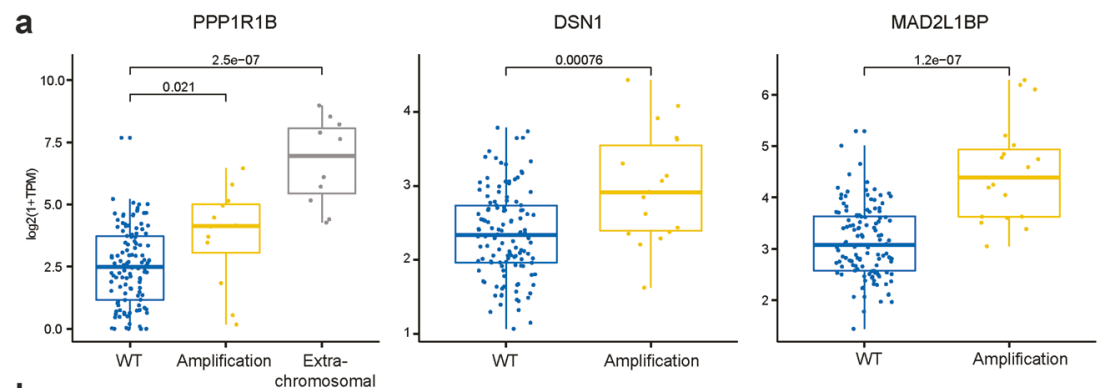

b

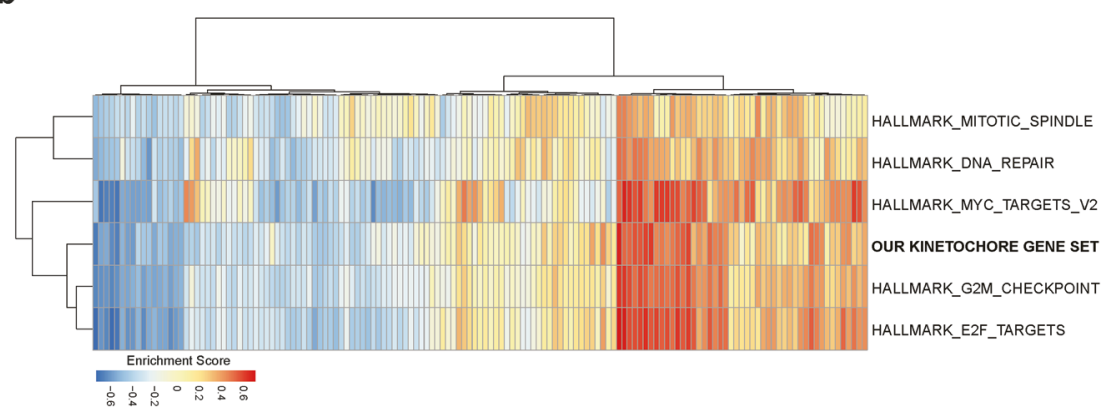

\section{The expression levels of inner and outer kinetochore components are altered in EAC cells}

The alterations in copy number and expression of our gene set highlighted by the WGS and RNA-seq analyses prompted us to investigate whether these changes were also reflected at protein level in BE and EAC cells by both Western blot and immunofluorescence microscopy. We analyzed the levels of a selection of SAC and kinetochore proteins, BubR1, CENP-C, Dsn1, Ndc80, and Spc24, in unsynchronized BE and EAC cells by Western blot (Fig. 6a). CPA cells were used as reference controls because are not dysplastic and have very few copy number changes [27] (Supplementary Table S1). In agreement with our WGS and RNA-seq results, Western blot analysis revealed substantial differences in the levels of kinetochore proteins both within individual cell lines and comparatively across the different $\mathrm{BE}$ and EAC cell lines. The levels of the outer kinetochore components Ndc80 and DSN1 (member of the Mis12 complex) were increased between two- and four-fold in all cell lines compared to CPA (Fig. 6a). The levels of the outer kinetochore component Spc24 (member of the Ndc80 complex) and inner kinetochore component CENP-C showed a more modest increase only in FLO and JH-Eso-Ad1 cells (Fig. 6a). By contrast, the mitotic checkpoint protein BubR1 showed slightly decreased expression in CPD, FLO, and JH-Eso-Ad1 cells. Interestingly, an additional, slower migrating BubR1 band was present in CPD and, more weakly, FLO cells, which could represent either a longer isoform or a specific post-translational modification (Fig. 6a).

We next analyzed the accumulation of kinetochore proteins in mitotic cells by immunofluorescence. The level of each kinetochore protein was normalized to the level of a centromeric marker derived from human CREST patient serum [21] (see Methods). The accumulation of Ndc80, Spc24, and BubR1 at kinetochores followed in large part the same profiles observed in our Western blot analysis (Fig. 6b-d). Ndc80 intensity was significantly higher in CPD and FLO cells than in CPA cells, but no significant difference was observed in JH-Eso-Ad1 cells (Fig. 6b). By contrast, accumulation of the other Ndc80 complex subunit, Spc24, was only reduced in CPD cells, but not in EAC cells, compared to CPA controls (Fig. 6c). In full accordance with the Western blot analysis, BubR1 levels were reduced in both EAC cell lines (Fig. 6d). Unfortunately, we failed to obtain a clear BubR1 signal in CPD cells, which might be possibly related to the presence of an additional band in Western blots (Fig. 6a). In contrast to the Western blot analysis, the levels of the Mis12 subunit DSN1 were significantly reduced in all cell lines (Fig. 6e). We then analyzed the levels of AURKB because of its key role in correcting improper chromosome attachments $[24,28]$. AURKB levels were strongly reduced in both CPD and FLO cells, but not in JH-Eso-Ad1 (Fig. 6f). Finally, comparison between mitoses with aligned or scattered chromosomes revealed that the levels of all these kinetochore proteins were significantly reduced on kinetochores of scattered chromosomes in almost all EAC cell lines, with the only exception of BubR1 in JH-Eso-Ad1 cells (Fig. 6b-f).

In conclusion, these results indicate that both inner and outer kinetochore proteins show different expression and kinetochore accumulation levels in dysplastic BE and EAC cells compared to CPA controls, which is likely to contribute to the defects in chromosome attachments observed in these cells. 


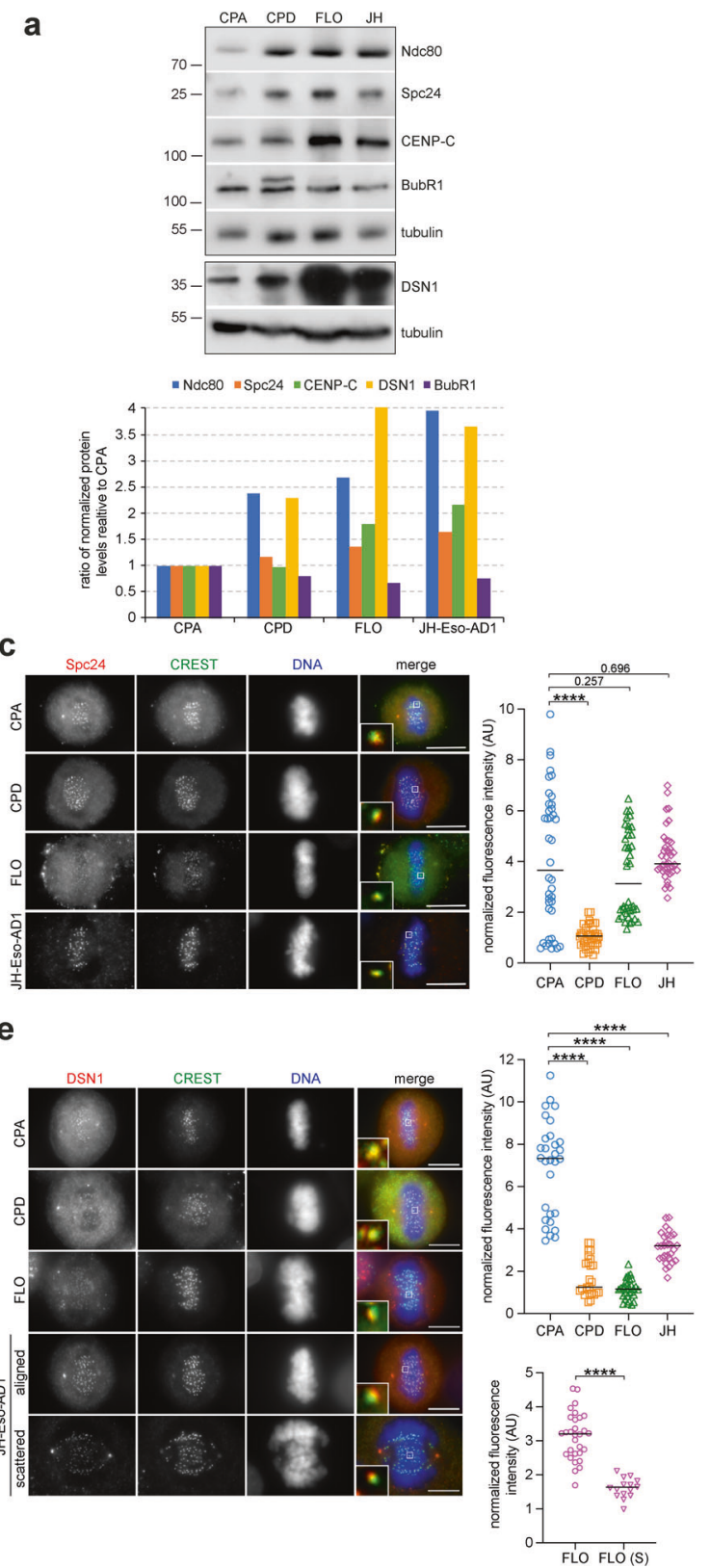

Fig. 6 Kinetochore protein levels are altered in dysplastic BE and EAC cells. a Analysis of protein expression in BE (CPA and CPD) and EAC cells (FLO and JH-Eso-AD1). Proteins extracts from the indicated BE and EAC cells were separated by SDS PAGE and analyzed by Western blot to identify the proteins indicated to the right. The numbers on the left indicate the sizes of the molecular mass marker. The graph at the bottom shows the quantification of protein levels, normalized to tubulin and relative to levels in CPA cells. b-f Indicated $\mathrm{BE}$ and EAC cells were fixed and stained to detect the

\section{EAC cells with scattered chromosomes have a significant increase of lateral kinetochore- microtubule attachments}

The initial lateral attachments of kinetochores to spindle microtubules are subsequently converted into end-on
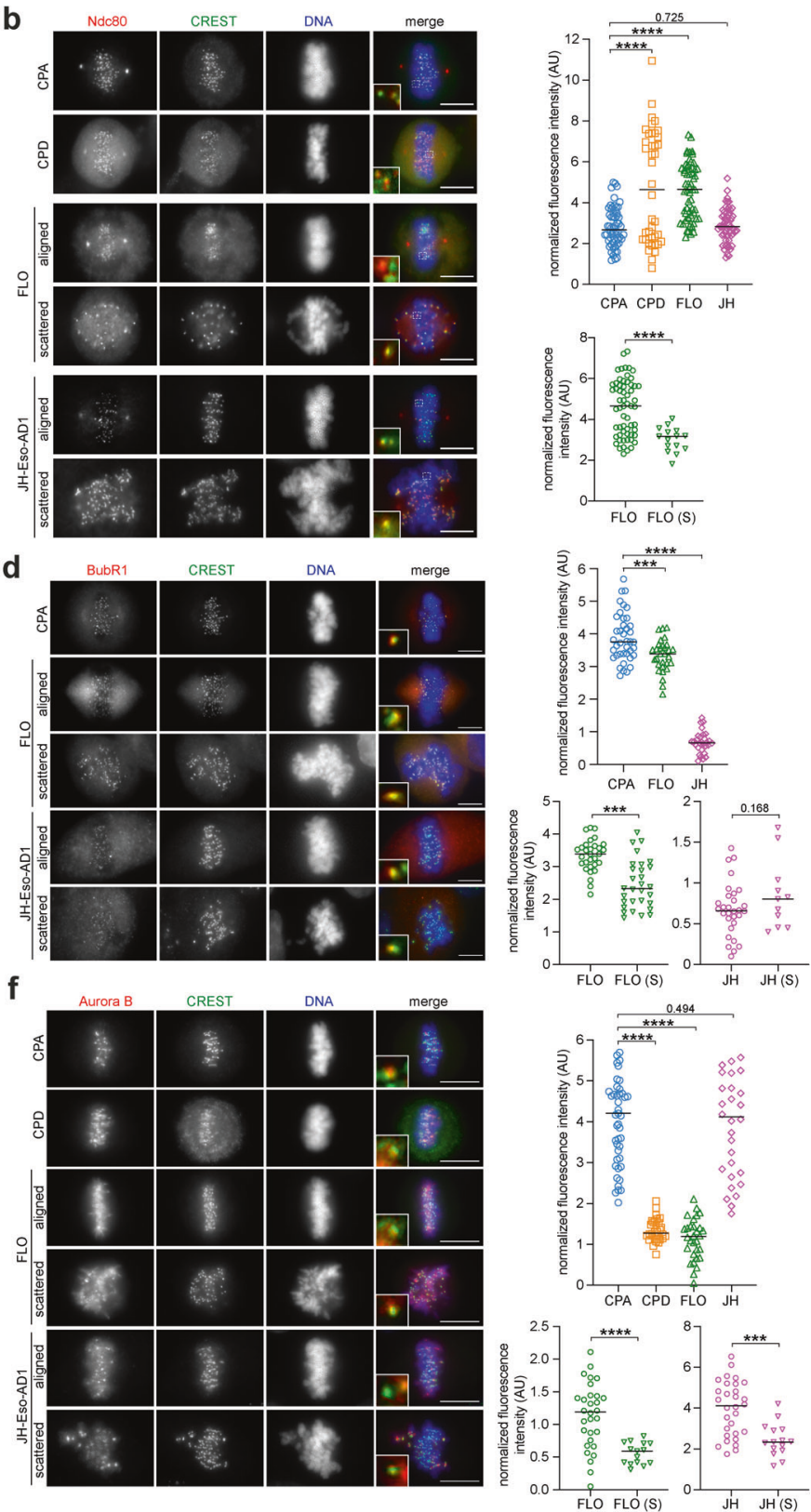

indicated epitopes. The insets show a $5 \times$ magnification of selected kinetochores. Bars, $10 \mu \mathrm{m}$. In each panel, the graphs to the right show quantification of fluorescence levels normalized to the centromere marker CREST (see Methods). More than 25 kinetochores from cells with aligned chromosomes and at least 10 kinetochores from cells with scattered (S) chromosomes were counted in $n \geq 3$ independent experiments. Horizontal bars indicate medians; $* * * * p<0.0001, * * * p$ $<0.001$ (two-tailed unpaired student's T-test).

attachments [29], which are necessary to impart the pushing and pulling forces required for chromosome congression [30]. The astrin-SKAP complex is recruited to mature, end-on kinetochores and is therefore used as a marker of end-on conversion [31]. To investigate whether the chromosome congression defects observed in EAC cells could 

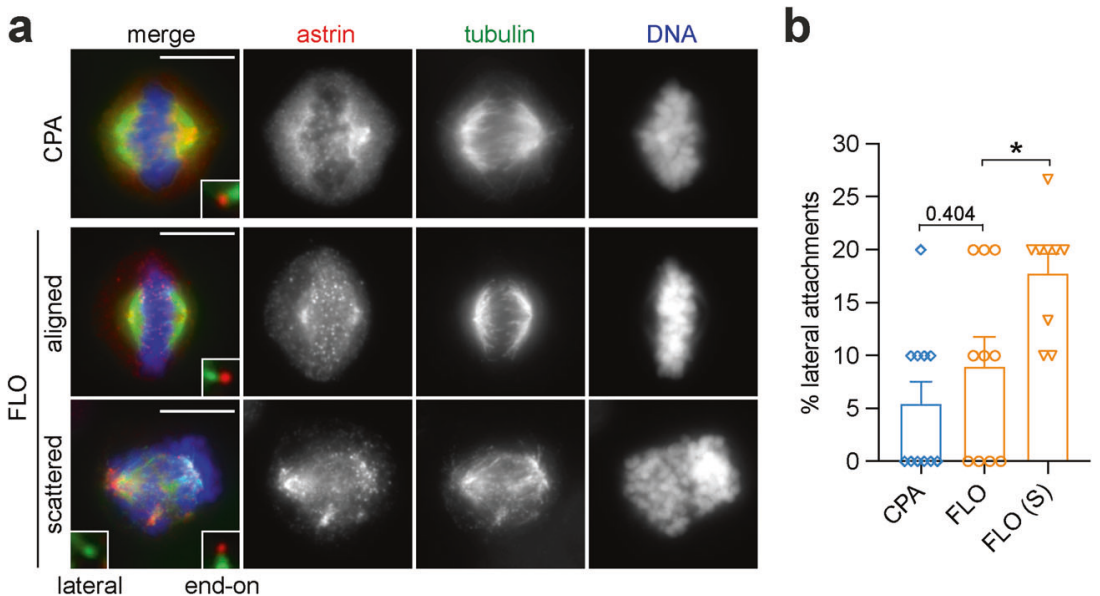

Fig. 7 FLO cells with scattered chromosomes display an increase in lateral kinetochore-microtubule attachments. a CPA and FLO cells were fixed and stained to detect the indicated epitopes. The insets show a $5 \times$ magnification of selected kinetochores. Bars, $10 \mu \mathrm{m}$. b Graph showing the quantification of lateral kinetochore-microtubule

result from a failure in converting lateral to end-on attachments, we stained with antibodies against astrin to visualize and quantify the different types of kinetochore-microtubule attachments (Fig. 7a). In both CPA and FLO cells that had successfully congressed to the metaphase plate, $<10 \%$ of kinetochores were laterally attached (Fig. 7b), whereas there was a two-fold increase of lateral attachments in FLO cells with scattered chromosomes (Fig. 7b). This data suggests that a problem in converting from lateral to end-on attachments could be in part responsible for chromosome congression failure in EAC cells.

\section{Discussion}

The emerging evidence that polyploidy contributes to cancer evolution and heterogeneity by promoting CIN highlights the need to study the mechanisms that cause genome doubling in cancers not only to understand its role in cancer biology, but also to aid in the classification and design of therapeutic treatments of highly heterogeneous cancers. In this study, we present evidence that polyploidy in EAC originates from mitotic slippage caused by defects in chromosome attachments during mitosis. Importantly, the frequency of these defects, $<10 \%$ in cells and around $20 \%$ in organoids (Figs. 1 and 3), is high enough to promote CIN, but not to significantly affect the viability of the entire cell population. We propose that these defects may permit the evolution of small clones within the large EAC cell population that can then more successfully adapt to the selective pressure of changing conditions.

The most prevalent mitotic defect observed in EAC cells was chromosome congression failure characterized by the attachments in CPA and FLO cells. 100 kinetochores were counted in each group; $n=11$ independent CPA cells, $n=10$ independent FLO cells and $n=9$ independent FLO cells with scattered chromosomes (S). Bars indicate standard errors; $* p<0.05$ (Mann-Whitney $U$ test).

presence of misaligned and scattered chromosomes, which is a phenotype typically caused by defective kinetochores invoking a robust SAC. Structural and/or functional alterations of kinetochore and centromeric proteins have been widely implicated in the promotion of chromosome mis-segregation and aneuploidy [32, 33]. WGS and RNAseq analyses coupled with analysis of kinetochore protein expression by Western blot and immunofluorescence revealed a number of copy number alterations and abnormal levels of numerous important constituents of the inner and outer kinetochore (Fig. 8). Notably, our findings highlighted clear imbalances in the abundance of kinetochore proteins in BE and EAC cells. For example, both Western blot and immunofluorescence analyses indicated that the stoichiometry of the Ndc80 complex subunits Ndc80 and Spc24 was altered, as in most cases Ndc80 was more abundant than Spc24 in CPD and EAC cells (Fig. 6). Ndc80 overexpression has previously been described in brain, liver, breast, and lung cancers and increase in total Ndc80 translated into increased accumulation of this protein at the kinetochore in human colorectal carcinoma (HCT116), colorectal adenocarcinoma (HT29), osteosarcoma (U2OS) and cervix adenocarcinoma (HeLa) cells [34, 35]. These findings led to speculate that increased accumulation of $\mathrm{Ndc} 80$, as part of the Ndc80 complex, might influence the interaction between kinetochores and microtubules in cancer cells. Our findings also support a similar conclusion that an increase of Ndc80 in EAC cells might be responsible for the chromosome alignment defects observed in these cells (Figs. 1 and 2). In addition, an increase in $\mathrm{Ndc} 80$ was associated with a decrease in AURKB in both CPD and FLO cells (Figs. 6 and 8). The combination of reduced AURKB and Ndc80 overexpression could result in low 
Fig. 8 Alteration in genes and proteins involved in chromosome attachments identified in this study.

Schematic diagram illustrating the different kinetochore layers and summarizing the different type of alterations for each specific gene and corresponding protein found in our study.

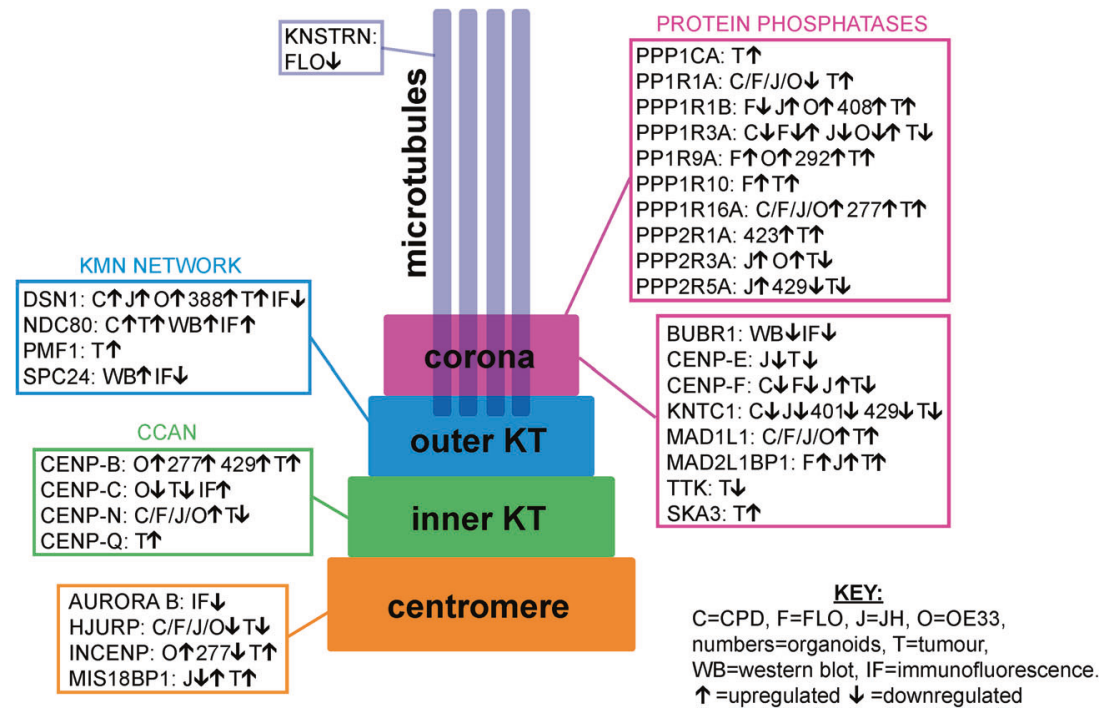

Ndc80 phosphorylation and the formation of hyperstable kinetochore-microtubule attachments and consequent problems in chromosome congression. Furthermore, the reduced levels of AURKB and BubR1 might weaken the SAC and allow mitotic slippage. It is important to point out that these alterations cause subtle changes in the regulation and mechanics of chromosome dynamics, which would allow most mitoses to progress normally. However, in a few cases these changes could lead to polyploidy and CIN, thereby promoting the evolution and diversification of new clones with potentially advantageous characteristics. Moreover, chromosome segregation defects could also in turn lead to additional changes in the copy number and expression of other mitotic and non-mitotic genes creating a self-feeding loop that would further refine the carcinogenic potential and adaptability of the clones over time and in response to specific selective pressures.

Our findings also indicated that the Mis12 component Dsn1 was amplified and expressed at high levels in both EAC cultured cells and tumors, but paradoxically its accumulation at kinetochore in mitosis was reduced (Figs. 4, 6, and 8). We don't have any explanation for this discrepancy at the moment, but these findings clearly indicate that a combination of different approaches-ranging from the analysis of genomes, transcriptomes, and proteomes down to the structure and composition of individual sub-cellular components-is necessary to fully evaluate the potential impact of gene and protein alterations in cancers.

One question raised from our data is whether the scattered chromosome phenotype occurs just because of chromosome congression failure or also because of cohesion fatigue after a prolonged mitotic arrest. The evidence that EAC cells take significantly longer than CPA to align their chromosomes (Fig. 2) would suggest problems in chromosome congression, although we cannot exclude that EAC cells take longer to align their chromosomes because they are polyploid. Moreover, our time-lapse experiments indicated that some EAC cells showed problems in maintaining chromosome alignment after mitotic arrest (Fig. 2 and Supplementary movies 3 and 4), which would suggest that the spread of the chromosomes over the mitotic spindle might also result from cohesion fatigue. Future time lapse experiments using multiple fluorescent markers, such as tubulin and cyclin B, could help resolve this issue.

Finally, our WGS analyses revealed that members of the PP1 family of phosphatases were frequently amplified in EAC cell lines, organoids, and tumors (Fig. 8). In particular, PPP1R1B, also known as DARPP-32, was one of the genes that showed the highest increase in copy number in EAC patients. DARPP-32 is a neuronal protein and a potent PP1 inhibitor [36]. Amplification of DARPP-32 and its truncated isoform t-DARPP has been found in $68 \%$ of gastric cancers and several studies indicated that it is also over-expressed in cancers of the breast, prostate, colon, and esophagusspecifically in $30 \%$ of esophageal squamous cell carcinomas [37-43]. PP1 phosphatases antagonize the phosphorylation of many mitotic proteins by AURKB [44] and it is therefore tempting to speculate that their de-regulation could affect kinetochore-microtubule attachments. Understanding how the PP1-AURKB regulatory balance is altered in EAC could provide extremely valuable insights for future targeted cancer therapies.

\section{Materials and methods}

\section{Cell culture}

Cell lines are listed in Supplementary Table S1 along with their characteristics, genetic information, origin, and source. 
RPE1 cells were cultured in Dulbecco's Modified Eagle Medium Nutrient Mixture F12 (Life technologies) supplemented with $10 \%$ FBS (Sigma) and $1 \%$ pen/strep (ThermoFisher). CPA and CPD cells were cultured in Keratinocyte-SFM supplemented with $2.5 \mu \mathrm{g}$ prequalified human Epidermal Growth Factor 1-53 (EGF) (Life technologies), $25 \mathrm{mg}$ Bovine Pituitary Extract (BPE) (Life technologies) and $0.5 \%$ penicillin/streptomycin (pen/strep) (ThermoFisher). FLO cells were cultured in Dulbecco's Modified Eagle Medium (DMEM) (Sigma) supplemented with $10 \%$ Fetal Bovine Serum (FBS) (Sigma) and 1\% pen/ strep (ThermoFisher). JH-Eso-AD1 cells were cultured in Minimum Essential Medium (MEM) (Sigma) supplemented with $10 \%$ FBS and $1 \%$ pen/strep (ThermoFisher). OE33 and OE19 cells were cultured in Roswell Park Memorial Institute (RPMI) Medium (Life technologies) supplemented with $10 \%$ FBS (Sigma) and 1\% pen/strep (ThermoFisher). All cells were cultured in a humidified atmosphere with $5 \%$ $\mathrm{CO}_{2}$ at $37^{\circ} \mathrm{C}$.

\section{Organoid culture}

Organoids are listed in Supplementary Table S4 along with their characteristics and genetic information. Derivation and culture of organoids were recently described [20]. Briefly, for passaging organoids were washed with PBS, centrifuged, and then disassociated by using TrypLE (Invitrogen) at $37{ }^{\circ} \mathrm{C}$. The cell pellet was re-suspended in $7.5 \mathrm{mg} / \mathrm{ml}$ basement membrane matrix (Cultrex BME RGF type 2BME-2, Amsbio) and plated as 10-15 $\mu \mathrm{l}$ droplets. After BME-2 polymerized, complete media was added, and organoid culture left at $37^{\circ} \mathrm{C}$. All organoids have been passaged at least 25 times and grew for over 6 months. Organoids were evaluated with primary tumors at DNA, RNA, and histological level to confirm their origin.

\section{Fluorescence microscopy}

Cells were grown on microscope glass coverslips (MenzelGläser) and fixed in either PHEM buffer (60 mM PIPES, 25 mM HEPES pH 7, $10 \mathrm{mM}$ EGTA, $4 \mathrm{mM} \mathrm{MgCl}_{2}, 3.7 \%$ [v/v] formaldehyde) for $12 \mathrm{~min}$ at room temperature or in ice-cold methanol for $10 \mathrm{~min}$ at $-20^{\circ} \mathrm{C}$. For astrin staining, coverslips were first incubated for $5 \mathrm{~min}$ in pre-extraction buffer (60 mM PIPES pH 7.0, $25 \mathrm{mM}$ HEPES pH 7.0, $10 \mathrm{mM}$ EGTA, $4 \mathrm{mM} \mathrm{MgSO}_{4}, 0.5 \%$ [v/v] Triton X-100) and then fixed using PHEM buffer. After fixing, coverslips were washed three times for 10 min with PBS and incubated in blocking buffer (PBS, $0.5 \%$ [v/v] Triton X-100 and 5\% [w/ v] BSA) for $1 \mathrm{~h}$ at room temperature. Coverslips were incubated overnight at $4{ }^{\circ} \mathrm{C}$ with the primary antibodies indicated in the figure legends, diluted in PBT (PBS, 0.1\% [v/v] Triton X-100 and 1\% [w/v] BSA). The day after, coverslips were washed twice for $5 \mathrm{~min}$ in PBT, incubated with secondary antibodies diluted in PBT for $2 \mathrm{~h}$ at RT and then washed twice with PBT and once with PBS. Coverslips were mounted on SuperFrost Microscope Slides (VWR) using VECTASHIELD Mounting Medium containing DAPI (Vector Laboratories). Phenotypes were blindly scored by at least two people independently. Images were acquired using a Zeiss Axiovert epifluorescence microscope equipped with MetaMorph software.

Organoids were cultured on chamber slide for 7 days and washed twice with PBS. Cultures were fixed in 4\% PFA at room temperature, quenched with $100 \mathrm{mM}$ glycine-PBS for 10 min, permeabilized in PBS $+0.5 \%$ Triton X-100 for 20 min, followed by blocking buffer (PBS, $1 \%$ BSA [w/v]) for $1 \mathrm{~h}$, and then incubated at $4{ }^{\circ} \mathrm{C}$ with primary antibodies. After overnight incubation, organoids were washed and incubated with appropriate secondary antibody for $1 \mathrm{~h}$ at room temperature as indicated above. Coverslips were mounted using VECTASHIELD Mounting Medium containing DAPI for nuclei staining. Organoids were imaged using a Leica confocal microscope TCS SP5, Z stacks were taken at $1-\mu \mathrm{m}$ intervals and scored by two independent researchers. Images were processed by Volocity image analyze software (Perkin Elmer, version 6.3.0).

Fiji [45] was used to generate maximum intensity projections, which were adjusted for contrast and brightness and assembled using Photoshop. Fluorescence intensity values of kinetochore proteins were calculated using Fiji software and the following formula: $\left(I_{\mathrm{K}}-I_{\mathrm{B}}\right)-\left(I_{\mathrm{C}}-I_{\mathrm{B}}\right) /$ $\left(I_{\mathrm{C}}-I_{\mathrm{B}}\right)=\left(I_{\mathrm{K}}-I_{\mathrm{C}}\right) /\left(I_{\mathrm{C}}-I_{\mathrm{B}}\right), \quad$ where $\quad I_{\mathrm{K}}=$ kinetochore intensity, $I_{\mathrm{C}}=$ CREST intensity, and $I_{\mathrm{B}}=$ background intensity.

\section{Time-lapse imaging}

Cells were plated on an eight-well $\mu$ slide (Ibidi) in their appropriate growth media. Prior to recording, media was replaced with Leibovitz's L-15 media (ThermoFisher) containing $0.5 \mu \mathrm{M}$ SiR-DNA dye (SpiroChrome). Cells were incubated in the dark at $37^{\circ} \mathrm{C}$ for $20 \mathrm{~min}$ and then imaged on an Olympus IX83 with an LED illuminator (Spectra-X, Lumencor), XY automated stage (ASI) in a $37^{\circ}$ $\mathrm{C}$ incubation chamber (Digital Pixel) controlled using Micromanager freeware. Images were captured every $5 \mathrm{~min}$ for 100 frames with $100 \mathrm{~ms}$ brightfield and $20 \mathrm{~ms}$ Cy5 exposure times. Further processing was carried out using Fiji [45].

\section{Western blot}

Cells were centrifuged, resuspended in phosphate buffer saline (PBS) and then an equal volume of 2x Laemmli buffer was added. Samples were then boiled for $10 \mathrm{~min}$ and 
stored at $-20^{\circ} \mathrm{C}$. Proteins were separated by SDS PAGE and then transferred onto PVDF membrane (Immobilon-P) at $15 \mathrm{~V}$ for $1 \mathrm{~h}$. Membranes were blocked overnight at $4{ }^{\circ} \mathrm{C}$ in PBS $+0.1 \%(\mathrm{v} / \mathrm{v})$ Tween $(\mathrm{PBST})$ with $5 \%(\mathrm{v} / \mathrm{v})$ dry milk powder. After blocking, membranes were washed once with PBST and then incubated with the appropriate primary antibody diluted in PBST $+3 \%$ (v/v) BSA (Sigma) for $2 \mathrm{~h}$ at RT. Membranes were washed $3 \times 5 \mathrm{~min}$ in PBST and then incubated with HRPA-conjugated secondary antibodies in $\mathrm{PBST}+1 \% \mathrm{BSA}$ for $1 \mathrm{~h}$ at room temperature. After further $3 \times 5 \mathrm{~min}$ washes in PBST, the signals were detected using the ECL West Pico substrate (ThermoFisher) and chemiluminescent signals were acquired below saturation levels using a G:BOX Chemi XRQ (Syngene) and quantified using Fiji [45].

\section{Antibodies}

The following antibodies and dilutions for Western blot (WB) and immuno-fluorescence (IF) were used in this study: mouse monoclonal anti $\alpha$-tubulin (clone DM1A, Sigma, T9026; dilutions for WB 1:20000, for IF 1:2000), rabbit polyclonal anti- $\beta$-tubulin (Abcam, ab6046; dilutions for WB 1:5000, for IF 1:400), mouse monoclonal anti $\gamma$ tubulin (Sigma, GTU88; dilutions for IF 1:200), mouse monoclonal anti-cyclin B1 (Santa Cruz, clone GNS1, sc245; dilution for WB 1:2000), mouse monoclonal antiAurora B (clone AIM-1, BD Transduction Laboratories, 611082; dilutions for WB 1:2000, for IF 1:100), rabbit polyclonal anti-phospho-histone H3 pS10 (Merck, 06-570; dilution for WB 1:10000 for IF 1:500), rabbit polyclonal anti-BubR1 (Abcam, ab172518; dilution for WB 1:10000, for IF 1:500), rabbit polyclonal anti-DSN1 (Sigma, SAB2702119; dilution for WB 1:2000, for IF 1:100), human anti-CREST (Antibodies Incorporated, 15-234; dilution for IF 1:1000) rat polyclonal anti-Plk4 (kind gift from P. Almeida Coelho and D. M. Glover, University of Cambridge, UK; dilution for IF 1:1000), rabbit monoclonal anti-Spc24 (Abcam, ab169786; dilution for WB 1:2000, for IF 1:200), mouse monoclonal anti-Ndc80/Hec1 (Santa Cruz, sc-515550, dilution for WB 1:500, for IF 1:50), rabbit polyclonal anti-astrin (Novus Biologicals, NB100-74638, dilution for IF 1:100). Peroxidase and Alexa-fluor conjugated secondary antibodies were purchased from Jackson Laboratories and ThermoFisher, respectively.

\section{Computational and statistical analyses}

Mutation and copy number calls for different BE and EAC cell lines as published [19] were used for analysis. Copy number calling was performed by FREEC [46], as described [19]. Mutations were called by GATK (Broad Institute, MA, USA), as described [19]. Amplifications were defined as genes with $2 \times$ the median copy number of the host chromosome or greater.

To capture mutations and copy number alterations in EACs and primary organoids, we used WGS sequencing data of 379 EAC cases from the Oesophageal Cancer Clinical And Molecular Stratification (OCCAMS) consortium [9]. We used final calls available for these datasets, where Strelka [47] was applied for calling SNVs/small indels and ASCAT [48] for copy number alterations. Genes were tagged as amplified if ploidy adjusted $\mathrm{CN}>2$ and $<10$ or extrachromosomal-like if their ploidy adjusted $\mathrm{CN}>10$. Along with WGS data, matched RNA-seq for subset of cases was used for transcriptomic analysis. We used processed expression quantification measured as Transcripts per Million (TPM) for analysis [9]. Both genomic alterations and transcriptomic analysis were restricted to a set of annotated kinetochore-related genes (Supplementary Table S5). Only genes altered in at least 3\% of cases were reported. To check whether amplification correlate with expression, we compared expression of the target gene between cases without alterations and cases with amplification; level of significance was measured by non-parametric Wilcoxon test.

We ran a single sample-based gene set enrichment method to measure enrichment of annotated gene signatures (collection of genes). In this case, we used known annotated hallmark gene signatures [49] related to cell cycle along with our annotated kinetochore genes and measured their enrichment across all EAC cases with RNA-seq data available using GSVA [50].

Unless otherwise specified, Prism8 (GraphPad) and Excel (Microsoft) were used for statistical analyses and to prepare graphs.

\section{Data availability}

The WGS and RNAseq data of the EAC primary tumors and $\mathrm{BE}$ and EAC cell lines were published before and deposited at the European Genome-phenome Archive (EGA); accession numbers can be found in the relative publications cited in the text. The RNAseq and WGS data of the organoid lines have been deposited at the EGA under accession number EGAD00001006738.

Acknowledgements We are very grateful to D.M. Glover for allowing us free access to his microscopy facility, and to M.R. Przewloka and P. Almeida Coelho for helpful suggestions and antibodies. We thank L. Capalbo for critical reading of the manuscript.

Funding SJS was supported by an MRC Ph.D. studentship. Work in PPD lab is supported by a BBSRC grant (BB/R001227/1). Work in CL lab is supported by BBSRC project grant BB/R004137/1. The laboratory of RCF is funded by a Core Programme Grant from the Medical Research Council (RG84369). We thank the Human Research Tissue Bank, which is supported by the UK National Institute for Health Research (NIHR) Cambridge Biomedical Research Centre, from 
Addenbrooke's Hospital. Whole-genome sequencing was funded by a program grant from Cancer Research UK (RG81771/84119) to RCF.

Author contributions SJS performed experiments, organized, and analyzed data, and helped with the preparation of the manuscript; XL performed experiments with organoids; SJ analyzed WGS and RNAseq data; GD analyzed WGS data; CL helped with the time-lapse experiments; RCF supervised the study, analyzed data, and acquired funding; PPD designed the experiments, supervised the study, analyzed data, acquired funding, and wrote the paper. All authors discussed the results and commented on the manuscript.

\section{Compliance with ethical standards}

Conflict of interest The authors declare no competing interests.

Ethical approval This study was approved by the Institutional Ethics Committees (REC 10/H0305/1), and all participants gave written informed consent as part of the OCCAMS (Oesophageal Clinical And Molecular Stratification) consortium.

Publisher's note Springer Nature remains neutral with regard to jurisdictional claims in published maps and institutional affiliations.

Open Access This article is licensed under a Creative Commons Attribution 4.0 International License, which permits use, sharing, adaptation, distribution and reproduction in any medium or format, as long as you give appropriate credit to the original author(s) and the source, provide a link to the Creative Commons license, and indicate if changes were made. The images or other third party material in this article are included in the article's Creative Commons license, unless indicated otherwise in a credit line to the material. If material is not included in the article's Creative Commons license and your intended use is not permitted by statutory regulation or exceeds the permitted use, you will need to obtain permission directly from the copyright holder. To view a copy of this license, visit http://creativecommons. org/licenses/by/4.0/.

\section{References}

1. Heng HH, Bremer SW, Stevens JB, Horne SD, Liu G, Abdallah BY, et al. Chromosomal instability (CIN): what it is and why it is crucial to cancer evolution. Cancer Metastasis Rev. 2013;32:325-40.

2. Holland AJ, Cleveland DW. Boveri revisited: chromosomal instability, aneuploidy and tumorigenesis. Nat Rev Mol Cell Biol. 2009;10:478-87.

3. Ganem NJ, Godinho SA, Pellman D. A mechanism linking extra centrosomes to chromosomal instability. Nature. 2009;460:278-82.

4. Cosenza MR, Kramer A. Centrosome amplification, chromosomal instability and cancer: mechanistic, clinical and therapeutic issues. Chromosome Res. 2016;24:105-26.

5. Dewhurst SM, McGranahan N, Burrell RA, Rowan AJ, Gronroos E, Endesfelder D, et al. Tolerance of whole-genome doubling propagates chromosomal instability and accelerates cancer genome evolution. Cancer Discov. 2014;4:175-85.

6. Ganem NJ, Storchova Z, Pellman D. Tetraploidy, aneuploidy and cancer. Curr Opin Genet Dev. 2007;17:157-62.

7. Bielski CM, Zehir A, Penson AV, Donoghue MTA, Chatila W, Armenia J, et al. Genome doubling shapes the evolution and prognosis of advanced cancers. Nat Genet. 2018;50:1189-95.
8. Kuznetsova AY, Seget K, Moeller GK, de Pagter MS, de Roos JA, Durrbaum M, et al. Chromosomal instability, tolerance of mitotic errors and multidrug resistance are promoted by tetraploidization in human cells. Cell Cycle. 2015;14:2810-20.

9. Frankell AM, Jammula S, Li X, Contino G, Killcoyne S, Abbas S, et al. The landscape of selection in 551 esophageal adenocarcinomas defines genomic biomarkers for the clinic. Nat Genet. 2019;51:506-16.

10. Noorani A, Li X, Goddard M, Crawte J, Alexandrov LB, Secrier $\mathrm{M}$, et al. Genomic evidence supports a clonal diaspora model for metastases of esophageal adenocarcinoma. Nat Genet. 2020;52:74-83.

11. Consortium ITP-CAoWG. Pan-cancer analysis of whole genomes. Nature. 2020;578:82-93.

12. Gerstung M, Jolly C, Leshchiner I, Dentro SC, Gonzalez S, Rosebrock D, et al. The evolutionary history of 2,658 cancers. Nature. 2020;578:122-8.

13. Gregson EM, Bornschein J, Fitzgerald RC. Genetic progression of Barrett's oesophagus to oesophageal adenocarcinoma. Br J Cancer. 2016;115:403-10.

14. Ross-Innes CS, Becq J, Warren A, Cheetham RK, Northen H, O'Donovan M, et al. Whole-genome sequencing provides new insights into the clonal architecture of Barrett's esophagus and esophageal adenocarcinoma. Nat Genet. 2015;47:1038-46.

15. Agrawal N, Jiao Y, Bettegowda C, Hutfless SM, Wang Y, David $\mathrm{S}$, et al. Comparative genomic analysis of esophageal adenocarcinoma and squamous cell carcinoma. Cancer Discov. 2012;2:899-905.

16. Stachler MD, Taylor-Weiner A, Peng S, McKenna A, Agoston AT, Odze RD, et al. Paired exome analysis of Barrett's esophagus and adenocarcinoma. Nat Genet. 2015;47:1047-55.

17. Lopes CAM, Mesquita M, Cunha AI, Cardoso J, Carapeta S, Laranjeira $\mathrm{C}$, et al. Centrosome amplification arises before neoplasia and increases upon p53 loss in tumorigenesis. J Cell Biol. 2018;217:2353-63.

18. Musacchio A. The molecular biology of spindle assembly checkpoint signaling dynamics. Curr Biol. 2015;25:R1002-18.

19. Contino G, Eldridge MD, Secrier M, Bower L, Fels Elliott R, Weaver $\mathrm{J}$, et al. Whole-genome sequencing of nine esophageal adenocarcinoma cell lines. F1000Res. 2016;5:1336.

20. Li X, Francies HE, Secrier M, Perner J, Miremadi A, GaleanoDalmau N, et al. Organoid cultures recapitulate esophageal adenocarcinoma heterogeneity providing a model for clonality studies and precision therapeutics. Nat Commun. 2018;9:2983.

21. Tan EM, Rodnan GP, Garcia I, Moroi Y, Fritzler MJ, Peebles C. Diversity of antinuclear antibodies in progressive systemic sclerosis. Anti-centromere antibody and its relationship to CREST syndrome. Arthritis Rheum. 1980;23:617-25.

22. Cheeseman IM. The kinetochore. Cold Spring Harb Perspect Biol. 2014;6:a015826.

23. Cheeseman IM, Chappie JS, Wilson-Kubalek EM, Desai A. The conserved KMN network constitutes the core microtubule-binding site of the kinetochore. Cell. 2006;127:983-97.

24. Cimini D, Wan X, Hirel CB, Salmon ED. Aurora kinase promotes turnover of kinetochore microtubules to reduce chromosome segregation errors. Curr Biol. 2006;16:1711-8.

25. Musacchio A, Desai A. A molecular view of kinetochore assembly and function. Biology (Basel). 2017;6:5.

26. Yang M, Li B, Tomchick DR, Machius M, Rizo J, Yu H, et al. p31comet blocks Mad2 activation through structural mimicry. Cell 2007;131:744-55.

27. Palanca-Wessels MC, Barrett MT, Galipeau PC, Rohrer KL, Reid BJ, Rabinovitch PS. Genetic analysis of long-term Barrett's esophagus epithelial cultures exhibiting cytogenetic and ploidy abnormalities. Gastroenterology. 1998;114:295-304. 
28. Carmena M, Wheelock M, Funabiki H, Earnshaw WC. The chromosomal passenger complex (CPC): from easy rider to the godfather of mitosis. Nat Rev Mol Cell Biol. 2012;13:789-803.

29. Alexander SP, Rieder CL. Chromosome motion during attachment to the vertebrate spindle: initial saltatory-like behavior of chromosomes and quantitative analysis of force production by nascent kinetochore fibers. J Cell Biol. 1991;113:805-15.

30. Skibbens RV, Skeen VP, Salmon ED. Directional instability of kinetochore motility during chromosome congression and segregation in mitotic newt lung cells: a push-pull mechanism. J Cell Biol. 1993;122:859-75.

31. Shrestha RL, Conti D, Tamura N, Braun D, Ramalingam RA, Cieslinski K, et al. Aurora-B kinase pathway controls the lateral to end-on conversion of kinetochore-microtubule attachments in human cells. Nat Commun. 2017;8:150.

32. Yuen KW, Montpetit B, Hieter P. The kinetochore and cancer: what's the connection? Curr Opin Cell Biol. 2005;17:576-82.

33. Cimini D, Degrassi F. Aneuploidy: a matter of bad connections. Trends Cell Biol. 2005;15:442-51.

34. Ferretti C, Totta P, Fiore M, Mattiuzzo M, Schillaci T, Ricordy R, et al. Expression of the kinetochore protein Hecl during the cell cycle in normal and cancer cells and its regulation by the $\mathrm{pRb}$ pathway. Cell Cycle. 2010;9:4174-82.

35. Perez de Castro I, de Carcer G, Malumbres M. A census of mitotic cancer genes: new insights into tumor cell biology and cancer therapy. Carcinogenesis. 2007;28:899-912.

36. Greengard P, Allen PB, Nairn AC. Beyond the dopamine receptor: the DARPP-32/protein phosphatase-1 cascade. Neuron. 1999;23:435-47.

37. Belkhiri A, Zhu S, El-Rifai W. DARPP-32: from neurotransmission to cancer. Oncotarget. 2016;7:17631-40.

38. El-Rifai W, Frierson HF Jr., Moskaluk CA, Harper JC, Petroni GR, Bissonette EA, et al. Genetic differences between adenocarcinomas arising in Barrett's esophagus and gastric mucosa. Gastroenterology. 2001;121:592-8.

39. El-Rifai W, Smith MF Jr., Li G, Beckler A, Carl VS, Montgomery E, et al. Gastric cancers overexpress DARPP-32 and a novel isoform, t-DARPP. Cancer Res. 2002;62:4061-4.
40. Vangamudi B, Peng DF, Cai Q, El-Rifai W, Zheng W, Belkhiri A. t-DARPP regulates phosphatidylinositol-3-kinasedependent cell growth in breast cancer. Mol Cancer. 2010;9:240.

41. Ebihara Y, Miyamoto M, Fukunaga A, Kato K, Shichinohe T, Kawarada Y, et al. DARPP-32 expression arises after a phase of dysplasia in oesophageal squamous cell carcinoma. Br J Cancer. 2004;91:119-23.

42. Gu L, Waliany S, Kane SE. Darpp-32 and its truncated variant tDarpp have antagonistic effects on breast cancer cell growth and herceptin resistance. PLoS One. 2009;4:e6220.

43. Wang MS, Pan Y, Liu N, Guo C, Hong L, Fan D. Overexpression of DARPP-32 in colorectal adenocarcinoma. Int J Clin Pract. 2005;59:58-61.

44. Nasa I, Rusin SF, Kettenbach AN, Moorhead GB. Aurora B opposes PP1 function in mitosis by phosphorylating the conserved PP1-binding RVxF motif in PP1 regulatory proteins. Sci Signal. 2018;11:eaai8669.

45. Schindelin J, Arganda-Carreras I, Frise E, Kaynig V, Longair M, Pietzsch T, et al. Fiji: an open-source platform for biologicalimage analysis. Nat Methods. 2012;9:676-82.

46. Boeva V, Popova T, Bleakley K, Chiche P, Cappo J, Schleiermacher G, et al. Control-FREEC: a tool for assessing copy number and allelic content using next-generation sequencing data. Bioinformatics. 2012;28:423-5.

47. Saunders CT, Wong WS, Swamy S, Becq J, Murray LJ, Cheetham RK. Strelka: accurate somatic small-variant calling from sequenced tumor-normal sample pairs. Bioinformatics. 2012;28:1811-7.

48. Van Loo P, Nordgard SH, Lingjaerde OC, Russnes HG, Rye IH, Sun W, et al. Allele-specific copy number analysis of tumors. Proc Natl Acad Sci USA. 2010;107:16910-5.

49. Liberzon A, Birger C, Thorvaldsdottir H, Ghandi M, Mesirov JP, Tamayo P. The molecular signatures database (MSigDB) hallmark gene set collection. Cell Syst. 2015;1:417-25.

50. Hanzelmann S, Castelo R, Guinney J. GSVA: gene set variation analysis for microarray and RNA-seq data. BMC Bioinf. 2013;14:7. 